\title{
To Formulate a Selective Patient Safety-related Policy for a Tertiary Care Hospital
}

\author{
${ }^{1} \mathrm{~S}$ Singh, ${ }^{2}$ Shakti Kumar Gupta, ${ }^{3} \mathrm{~S}$ Arya, ${ }^{4} \mathrm{~V}$ Aggarwal
}

\begin{abstract}
Patient safety is the absence of preventable harm to a patient during the process of healthcare (WHO). Accuracy of patient identification remains a priority focus of healthcare organization. Identifying patients accurately presents many unique challenges in today's healthcare settings. We need to understand how human factors can be used to reduce adverse events. Using a human factor approach, the human system interface can be improved by providing better designed system and processes. This involves simplifying processes, standardizing procedures, providing back up when human fails, improving communication, redesigning equipment and engendering a consciousness of behavioral organization and technological limitation that lead to error. The above study was an initiative toward simplifying processes and standardizing procedures. It was a descriptive cross-sectional study carried out between April to August 2013. The tool used was a check list made after an exhaustive review of literature and validated by experts in quality assurance from NABH accredited private hospitals. The study population of 100 people which included doctors, nurses, paramedical staff and quality managers of tertiary care public and private hospitals were approached for interaction against the back drop of the check list. Response rate was $61 \%$. Policy was framed after incorporating inputs from responses received against the back drop of the check list.
\end{abstract}

Keywords: Patient identification policy, Patient safety.

How to cite this article: Singh S, Gupta SK, Arya S, Aggarwal V. To Formulate a Selective Patient Safety-related Policy for a Tertiary Care Hospital. Int J Res Foundation Hosp Healthc Adm 2014;2(2):94-102.

Source of support: Nil

Conflict of interest: None

\section{INTRODUCTION}

The issue of human errors in complex systems has been a topic of debate for decades. In the aviation industry system

\footnotetext{
${ }^{1}$ Senior Resident, ${ }^{2}$ Medical Superintendent, ${ }^{3}$ Professor ${ }^{4}$ General Manager

1,3 Department of Hospital Administration, All India Institute of Medical Sciences, New Delhi, India

${ }^{2} \mathrm{Dr}$ RP Centre for Ophthalmic Sciences, All India Institute of Medical Sciences, New Delhi, India

${ }^{4}$ Max Hospital, New Delhi, India
}

Corresponding Author: S Singh, Senior Resident, Department of Hospital Administration, All India Institute of Medical Sciences, New Delhi, India, e-mail: sheetalsingh2003@ hotmail.com failures cause great publicity and are addressed quickly for a very simple reason: aviation accidents attract great attention because they involve a large number of people and resources. Accidents and complications are common in health care as well, and can be deadly. However, they happen one or two at a time and are far less dramatic. Only in the 1990s did errors in healthcare settings begin to draw public attention. ${ }^{1}$ Patient safety is an essential and vital component of quality care. Healthcare providers face many challenges in today's healthcare environment in trying to keep patients safe. Patient safety is a discipline in the healthcare sector that applies safety science methods toward the goal of achieving a trustworthy system of healthcare delivery. ${ }^{2}$ Patient safety is also an attribute of healthcare systems; it minimizes the incidence and impact of, and maximizes recovery from, adverse events. ${ }^{3,4}$

In 1999, the Institute of Medicine (IOM) described the healthcare system as fractured, prone to errors, and detrimental to safe patient care. It defined patient safety as freedom from accidental injury and further stated that ensuring patient safety involves the establishment of operational systems and processes that minimize the likelihood of errors and maximize the likelihood of intercepting them when they occur. ${ }^{5}$

\section{STRATEGIES TO IMPROVE PATIENT SAFETY²}

\section{Developing Protocols and Guidelines ${ }^{1}$}

One of the ways to avoid human error is to reduce reliance on short-term memory. This limited cognition should be used to perform only essential tasks. Checklists, guidelines, and reminders are successful tools for workers to ensure better patient communication. Simple examples, such as identification by color coding or eliminating drugs that sound or look alike have proved to be effective.

\section{Increasing Patients Involvement ${ }^{6}$}

Patients can play a key role in ensuring that healthcare is safe. ${ }^{6}$ Increasing patient awareness of safety issues is a powerful but underused strategy toward improving the quality of healthcare. With emerging consumerism, patients are better aware of problems that occur in hospitals; they become active players in the system. Increased patient awareness can be a crucial part of risk-prevention. ${ }^{7}$ 
To Formulate a Selective Patient Safety-related Policy for a Tertiary Care Hospital

\section{Leadership Commitment to Safety Culture ${ }^{1}$}

Leaders of healthcare organizations need to become patient safety champions and encourage an organizational culture where disclosing adverse events and investigating root causes is not only nonthreatening but also an organic part of the organizational process. Every organization should maintain a continuous focus on redesigning and improving safety systems, especially in the intensive care unit, emergency room, and operating room. Training on safety issues for medical personnel is also recommended. Hospital administrators must initiate change management programs to build support for patient safety and get leaders across the organization committed to a prevention program. ${ }^{8}$

\section{Educating Healthcare Providers on Safety Culture $^{9}$}

Hospitals play an important role in educating and training. On-site experience and continuing education for staff help establish positive attitudes toward preventing adverse events. Teaching hospitals have a responsibility to instruct residents regarding importance of patient safety and demonstrate how to scan for potential problems and intercept adverse events.

\section{Creating a Culture of Safety ${ }^{10}$}

a. Hospitals should refrain from assigning fault to individuals and foster the attitude that mistakes are a chance to learn and improve care.

b. Processes to improve quality should be established among hospitals. ${ }^{11}$

c. Patient safety should become part of performance assessment and accountability.

2014 joint commission national patient safety goals: Among numerous patient safety Goal one of the most important national patient safety Goal of 2014. is ${ }^{12}$

Improve the accuracy of patient identification.

Use at least two patient identifiers when providing care, treatment or services.

\section{PATIENT IDENTIFICATION POLICY FOR A TERTIARY CARE HOSPITAL}

To improve the accuracy of patient identification is one of the national patient safety goal given by Joint Commission 2012 and so it is one of the patient safety issues selected for formulating policy.

\section{Need for the Study}

Throughout the healthcare industry, the failure to correctly identify patients continues to result in medication errors, transfusion errors, testing errors, wrong person procedures, and the discharge of infants to the wrong families. Between November 2003 and July 2005, the United Kingdom National Patient Safety Agency reported 236 incidents and near misses related to missing wristbands or wristbands with incorrect information. The quantum of error in patient identification in our country is not clear because of lack of reliable and validated data. The problem of patient identification is compounded further because there are no standard guidelines on this subject. We should have standard policy for identifying patients in hospital. Hence, there is need for the conduct of this study.

\section{METHODOLOGY}

It was a descriptive cross-sectional study carried out between April to August 2013. The study population included Doctors, Nursing personnel, Paramedical staff and quality managers of tertiary care hospital from one public and two private hospitals. Check list was made after an exhaustive review of literature which was then improvised. Validation of the checklist was done by experts in quality assurance from NABH accredited private hospitals. Subsequently, interaction was done with study population against the back drop of the check list and patient identification policy was formulated.

\section{Analysis and Results}

One hundred people which included doctors, nurses, paramedical staff and quality managers of tertiary care public and private hospitals were approached for interaction against the back drop of the check list. Total response rate was $61 \%$. Seventeen doctors responded out of 25 doctors approached, 20 nurses responded out of 25, 21 quality managers interacted out of 25 and 11 paramedical responded out of 25 approached. Policy was framed after incorporating inputs from responses received against the back drop of the check list.

\begin{tabular}{lll}
\multirow{2}{*}{$\begin{array}{l}\text { Tertiary care } \\
\text { hospital }\end{array}$} & Quality operating process & Document no: \\
\cline { 2 - 3 } & Manual of operations & Date of issue: \\
\hline
\end{tabular}

\section{PATIENT IDENTIFICATION POLICY FOR A TERTIARY CARE HOSPITAL ${ }^{13}$}

\begin{tabular}{ll} 
Service name: & $\begin{array}{l}\text { Patient identification policy for a } \\
\text { tertiary care hospital }\end{array}$ \\
\hline Date Created: & $\begin{array}{l}\text { Name: } \\
\text { Signature: }\end{array}$ \\
Rpproved by: & $\begin{array}{l}\text { Name: } \\
\text { Signature: }\end{array}$ \\
Reviewed by: & $\begin{array}{l}\text { Name: } \\
\text { Signature: }\end{array}$ \\
Issued by: & $\begin{array}{l}\text { Name: } \\
\text { Signature: }\end{array}$ \\
\hline
\end{tabular}




\begin{tabular}{lll}
\multirow{2}{*}{$\begin{array}{ll}\text { Tertiary care } \\
\text { hospital }\end{array}$} & Quality operating process & Document no: \\
\cline { 2 - 3 } & Manual of operations & Date of issue: \\
\hline
\end{tabular}

\section{PURPOSE $^{14}$}

This policy provides guidance to the staff to make sure the correct identity of all patients at all times and to restrict the risk of misidentification and adverse outcomes of care.

This policy applies to all patients who are in receipt of care or treatment from tertiary care hospital healthcare staff. This involves the following patients:

- All in-patients (including those waiting in emergency department undergoing assessment and investigations prior to admission).

- Patients receiving treatment/consultation in outpatient department

- Patients attending day care units for treatment.

\section{SCOPE}

This policy is applicable to all members of staff who deal with patient care.

\section{Staff Includes}

- Clinicians/Surgeons

- Associated health professionals

- Nursing staff

- Phlebotomists

- Pharmacists

- Staff involved in the management of patient care through the booking and management of appointments, admissions, transfers and any other action where the identification of the patient must be accurate and valid. ${ }^{13}$

\begin{tabular}{lll}
\multirow{2}{*}{$\begin{array}{l}\text { Tertiary care } \\
\text { hospital }\end{array}$} & Quality operating process & Document no: \\
\cline { 2 - 3 } & Manual of operations & Date of issue: \\
\hline
\end{tabular}

\section{WHAT IS THE PROCESS FOR IDENTIFYING PATIENT WITH ID BAND? ${ }^{13}$}

- The hospital staff should make sure that all in-patients must wear ID Bands.

- Before carrying out any intervention or procedure, staff undertaking the intervention or procedure should confirm the patient's identity.

- No procedure or intervention should be performed if the patient has no ID band.

- Where it is essential to remove a patient's ID band, for whatever reason, it is the responsibility of the staff, removing the wristband to ensure that it is replaced without delay. If this is not possible, then they should make sure that the reason for removal is documented in the patient's records.

- At least two identifiers (e.g. patient's full name and date of birth) must be used to verify a patient's identity. Neither of these identifiers should be the patient's room number or location.

\section{Identifiers used for Identification of Patients in a Tertiary Care Hospital}

- First and last name of the patient spelt in full - Initials are not permitted ${ }^{15}$

- Tertiary care hospital - UHID

- Date of birth

- IP No.

\section{What are the Steps to Identify the Patients?}

There are three steps to identify patients. These should be undertaken in the following order (if the first is not possible, undertake the second, etc): ${ }^{16}$

- Ask patient's name and date of birth. Check this is wellmatched with the patient's ID band.

\begin{tabular}{lll} 
Tertiary care & Quality operating process & Document no: \\
\cline { 2 - 3 } hospital & Manual of operations & Date of issue: \\
& Patient identification policy & \\
\hline
\end{tabular}

- If the patient is incapable to tell their name or in case of comatose patients, refer to the ID band and, if possible confirm the information by asking family member, attendant or another member of the clinical staff who was taking care of the patient.

- Ask patient's relative/attendant to identify the patient by name and/or date of birth. Do not read out the name or date of birth to the attendant let the attendant/relative tell the name and date of birth of the patient.

\section{What are the Types of Identification Band?}

\section{There are two Types of Identification Bands}

1. White bracelet: For those patients who do not have any known allergies. ${ }^{13}$

The ID band must contain the readable information of the patient in black text on a white background:

\section{IN CASE PATIENT IS ADMITTED IN WARD}

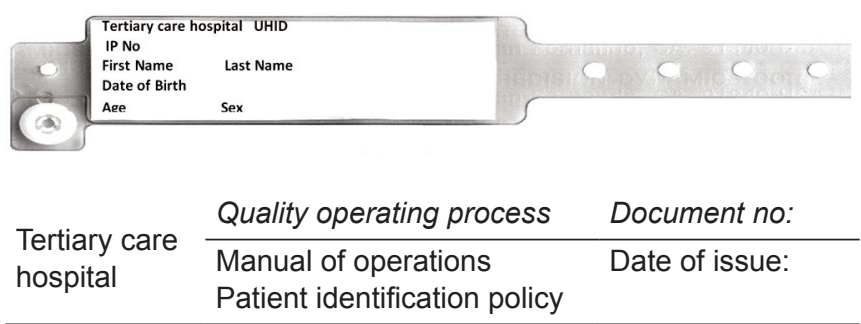


IN CASE PATIENT IS IN EMERGENCY DEPARTMENT

\section{Before Registration of the Patient - Temporary ID Band can be used}

\begin{tabular}{|ll|}
\hline ER No. & \\
First name & Last name \\
Date of birth & \\
Age & Sex \\
\hline
\end{tabular}

After Registration of Patient - Permanent ID Band

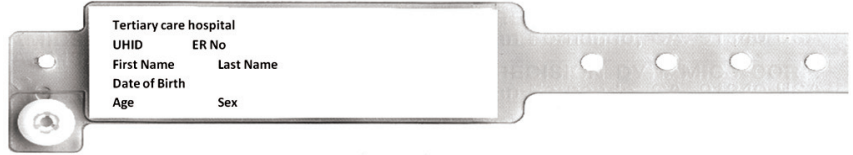

2. Red bracelet: Patients who have an allergy or gives history of allergy.

- All patients must be enquired if they are allergic to anything when they are admitted/treated. Note that an 'allergy' can include latex and other material components as well as medicines.

- Patients with a known allergy should wear an additional red identity band on the same wrist/ankle. This will act as a caution signal to staff to refer to the patient's medical notes for further details.

- Information to be recorded on the red band:
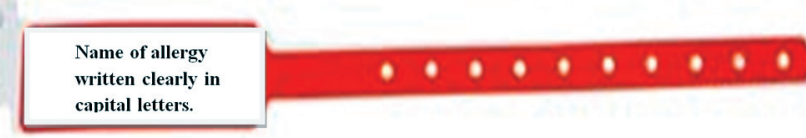

\begin{tabular}{lll}
\multirow{2}{*}{$\begin{array}{l}\text { Tertiary care } \\
\text { hospital }\end{array}$} & Quality operating process & Document no: \\
\cline { 2 - 2 } & Manual of operations & Date of issue: \\
& Patient identification policy & \\
\hline
\end{tabular}

\section{RESPONSIBILITIES OF THE STAFF ${ }^{17}$}

Director/Medical Superintendent/Nursing Superintendent: Responsible for ensuring that this policy is implemented operationally and monitored.

- Should ensure that staff in their areas are aware of the policy. Understand it and implement it.

- They should ensure that failures to comply with the policy are reported via the incident reporting system and the appropriate action is taken to prevent recurrence.

- Investigating failures to act in accordance with the policy and also ensure that corrective action is taken to prevent a recurrence.

\section{Responsibility of Clinicians/Surgeons}

- Any clinician/surgeon who removes an ID band (to perform a procedure/or examination) should ensure that another one is applied.
- The clinician before performing any treatment should check the ID band beforehand to ensure the correct patient.

- They should check the patient's identity and a signed consent form before commencing surgery.

\section{Clinical and Nonclinical Staffs should}

- Maintain the standards in this policy and accept accountability for their practice.

- Report all failures to comply with the policy via the risk incident system.

- Ensure that patients are identified using accurate personal details.

- Ensure that all patient details entered by them onto the ID bands are valid and accurate.

- Ensure that any ID band removed by them (or where more appropriate, another member of staff) is replaced without delay and that the information on the replacement ID band is valid and accurate.

\begin{tabular}{lll}
\multirow{2}{*}{$\begin{array}{l}\text { Tertiary care } \\
\text { hospital }\end{array}$} & Quality operating process & Document no: \\
\cline { 2 - 3 } & Manual of operations & Date of issue: \\
& Patient identification policy & \\
\hline
\end{tabular}

If immediate replacement is not possible then it is the responsibility of the member of staff to make clear options for the patient's correct identification.

\section{Staff at Central Admission Office}

- Ensure that correct patient details are entered at the time of registration.

- Validating the information given by the patient and/or their career/relative.

Any member of staff if discovers that a patient does not have an ID band then he/she has to assume responsibility for correctly identifying them. In general it will be nursing staff that will be responsible for the generation and application of the patient ID band. However, it is the responsibility of all staff to check the validity and correctness of patient identification before carrying out any actions relating to patient care.

\section{WHERE IDENTIFICATION OF PATIENT IS MUST? ${ }^{18}$}

The list below is not exclusive. Patients should always wear an identification band.

- Blood sampling

- Blood transfusion

- Collecting of patient bodily fluid samples

- Confirmation of death

- Administration of all medicines

- Surgical intervention and any invasive procedure 
- Transport/transfer of the patient

- X-rays and imaging procedures

\begin{tabular}{|c|c|c|}
\hline $\begin{array}{l}\text { Do not proce } \\
\text { The ID band }\end{array}$ & $\begin{array}{l}\text { Alert! } \\
\text { ust be replaced by the nurse } \\
\text { before the procedure can }\end{array}$ & $\begin{array}{l}\text { ent has no ID band } \\
\text { aring for the patient } \\
\text { gin }\end{array}$ \\
\hline & Quality operating process & Document no: \\
\hline hospital & $\begin{array}{l}\text { Manual of operations } \\
\text { Patient identification policy }\end{array}$ & Date of issue: \\
\hline
\end{tabular}

\section{WHEN TO PUT ID BAND?}

\section{Patients Admitted in a Hospital}

All in-patients (including those waiting in the emergency department undergoing assessment and investigations prior to admission) must wear an identification (ID) band for safety purposes unless patient refuses to wear one or it is contrary to the patient's well-being.

The ID band must be applied to patient on admission to hospital, or once the patient has entered a department for treatment by the assigned nurse.

\section{ID BAND SHOULD ALSO BE APPLIED TO ${ }^{8}$}

- Patients in the emergency department who have been through triage and are receiving treatment

- Patients in the day care

The ID band should be worn on the dominant arm that is the side used for writing, it is then less likely to be removed when for example, intravenous access lines are inserted.

The ID band must have the following information:

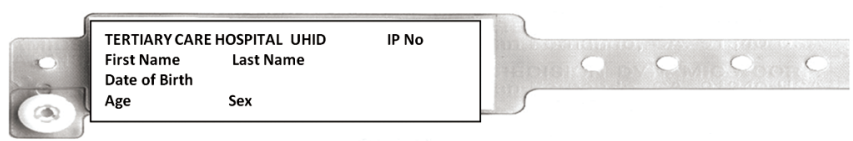

\begin{tabular}{c} 
The patient's room number or location should never be used for \\
identification \\
$\begin{array}{c}\text { The initials of patient's name should never be used for patient } \\
\text { identification }\end{array}$ \\
\hline
\end{tabular}

\begin{tabular}{lll}
\multirow{2}{*}{$\begin{array}{l}\text { Tertiary care } \\
\text { hospital }\end{array}$} & Quality operating process & Document no: \\
\cline { 2 - 3 } & Manual of operations & Date of issue: \\
\hline
\end{tabular}

\section{WHEN PATIENT IS TRANSFERRED WITHIN THE HOSPITAL ${ }^{19}$}

When patient is transferred within the hospital the staff who receives the patient shall check the patient wristband, with the patient, if the patient is capable to do so, along with the medical record for positive patient identification.

\section{When the Patient is Transferred from other Hospital}

Patients who are transferring in from other hospitals will have a tertiary care hospital ID Band applied immediately on arrival.

\section{In Case ID Band is Damaged}

If the patient ID band is removed, faded damaged or unreadable, a replacement ID band will be applied right away, by the assigned nurse who is taking care of the patient.

The ID band must not be removed until discharge procedure is completed

\section{DEPARTMENT OF EMERGENCY}

At the time of initial assessment by CMO and nurse, a temporary ID band will be applied to the patient by the assigned nurse. The ID band must contain the following clearly written, legible information of the patient in black text on a white background:

\section{Temporary ID Band which is Applied before Registration of the Patient}

\begin{tabular}{|ll|}
\hline ER No & \\
First name & Last name \\
Date of birth & \\
Age & Sex \\
\hline
\end{tabular}

Temporary band should be replaced by permanent ID Band by assigned nurse after the registration of the patient.

\begin{tabular}{lll}
\multirow{2}{*}{$\begin{array}{l}\text { Tertiary care } \\
\text { hospital }\end{array}$} & Quality operating process & Document no: \\
\cline { 2 - 3 } & Manual of operations & Date of issue: \\
\hline
\end{tabular}

\section{After Registration of the Patient-Permanent ID Band}

The band will contain the following information:

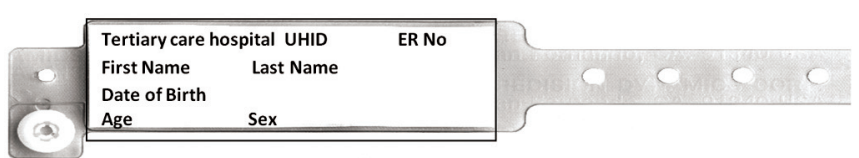

In case of allergy, the patient in emergency department should wear additional red band along with white colored ID Band specifying the name of allergy

\section{Transfer of a Patient from Emergency Department to Ward}

The staff receiving the patient shall check the patient's ID band with the patient, if the patient has the capacity to 
do so, along with the medical records for positive patient identification. The ID band will be changed to identify which ward the patient has been admitted.

\section{SPECIFIC SITUATIONS}

\section{Uncomprehending Patients}

Patients who are incapable of confirming their own identity (too young, unconscious, incoherent, language difficulties). An accompanying adult must answer on behalf of them.

\section{Language Problem}

An interpreter must be used if there is language problem. In case interpreter is not available in hospital the interpreter should be contacted on phone.

\begin{tabular}{lll}
\multirow{2}{*}{$\begin{array}{l}\text { Tertiary care } \\
\text { hospital }\end{array}$} & Quality operating process & Document no: \\
\cline { 2 - 3 } & Manual of operations & Date of issue: \\
\hline
\end{tabular}

\section{Unknown and Unconscious Patients}

In the event of unknown and unconscious patients (such as trauma patients), and where there is no possibility of positively identifying the patient. The identification is made by means of a band which must have the following information:

\begin{tabular}{|l|}
\hline Unknown male/female \\
Date of arrival \\
Time of arrival
\end{tabular}

Along with the ID band, preferably the photograph of the patient should be taken and the print out should be kept in patient record for identification purpose

\section{Patient with Missing Limbs}

If a limb is missing then bands must be securely attached to the patients clothing using strapping, on an area of the body which is clearly visible. The band must be reattached as clothing is changed, and must accompany the patient at all times. In emergency or operative situations where clothing is removed, identification must be attached to the patient's skin using see-through plastic adhesive film.

\section{Deceased Patients}

All deceased patients MUST be correctly identified with 2 identification bands - one attached to the wrist and one attached to the ankle. If a limb(s) is missing then attach one label to an available limb and the other to the patient's skin using transparent tape.

\begin{tabular}{|lll|}
\hline Tertiary care hospital & UHID & ER No./IP No. \\
First name & Last name & \\
Date of birth & \\
Age & Sex & \\
Religion (if known) & & \\
\hline
\end{tabular}

\begin{tabular}{lll}
\multirow{2}{*}{$\begin{array}{ll}\text { Tertiary care } \\
\text { hospital }\end{array}$} & Quality operating process & Document no: \\
\cline { 2 - 3 } & Manual of operations & Date of issue: \\
& Patient identification policy & \\
\hline
\end{tabular}

\section{Theater/Sedated Patients}

Patient identification is confirmed by theater staff prior to being anesthetized according to operating theater procedures.

Where the ID band is attached to the patient's wrist it will compromise patient safety hence ID band should be applied to the patient's ankle by the assigned nurse before shifting patient to theater.

\section{Extreme Emergencies}

Clinical care may take priority over attaching an ID band to the patient in extreme emergencies and possibly lifethreatening situations. The nurse responsible for patient care at the point of time must take appropriate steps to identify the patient using the hospital number and/or the ED number. Once the surname, forename, date of birth, gender and hospital number are confirmed, a new ID band must be attached to the patient immediately.

\section{NEONATES}

Color coded ID band are used for Babies identification. Blue colored ID band are used for male and pink for female infant.

Two ID band should be applied to two separate baby limbs by the assigned nurse immediately after the birth. Two people (either a doctor and nurse or nurse $\&$ hospital attendant) will accompany the baby from OT/Labor room to NICU/Nursery. The minimum information mentioned on the ID bands is mentioned below:

\begin{tabular}{lll}
\multirow{2}{*}{$\begin{array}{l}\text { Tertiary care } \\
\text { hospital }\end{array}$} & Quality operating process & Document no: \\
\cline { 2 - 3 } & Manual of operations & Date of issue: \\
& Patient identification policy & \\
\hline
\end{tabular}

Male

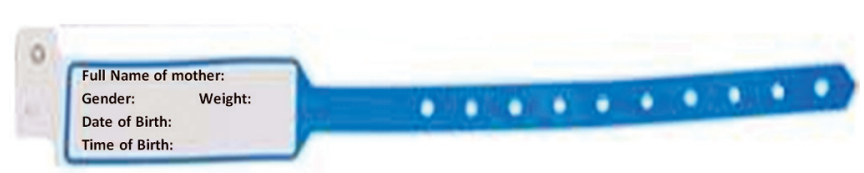

\section{Female}

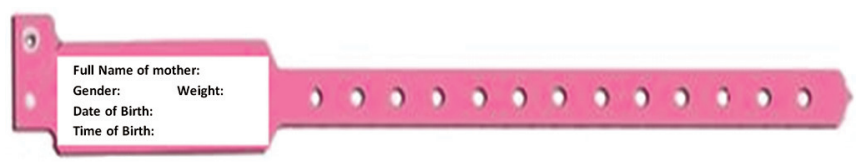

\section{Additional Identifier}

A color coded card should be attached to the baby's warmer/ cot displaying the following information. 


\begin{tabular}{|c|c|c|}
\hline \multicolumn{3}{|l|}{$\begin{array}{l}\text { Date of birth: } \\
\text { Time of birth: }\end{array}$} \\
\hline \multirow{2}{*}{$\begin{array}{l}\text { Tertiary care } \\
\text { hospital }\end{array}$} & Quality operating process & Document no: \\
\hline & $\begin{array}{l}\text { Manual of operations } \\
\text { Patient identification policy }\end{array}$ & Date of issue: \\
\hline
\end{tabular}

\section{In Case of Twins, ${ }^{20}$}

Twins should be named as Twin 1 and 2 .

The following identifiers must be included in the babies' ID bands.

\begin{tabular}{|llll|}
\hline \multicolumn{2}{|l|}{ Twin 1 } & \multicolumn{2}{l|}{ Twin 2 } \\
Full name of mother & Full name of mother \\
Time of birth & Date of birth & Time of birth & Date of birth \\
Gender & Weight & Gender & Weight \\
\hline
\end{tabular}

\section{MOTHER}

After delivery of the baby, mother to be given an additional ID band with the following baby's details.

Full name of mother:
Gender: Weight:
Date of birth:
Time of birth:

\section{OUTPATIENT DEPARTMENT}

a. Registration: In the outpatient clinic setting, verification of the patient will be made by the staff posted at registration when the patient arrives and get him/her self registered. Staff posted at registration counter will ask the patient or their care taker/relative to state:

- Patient's full name

- Father name

- In case of females father name/husband names

- Patient's date of birth

- Age

- Gender

- Patient's address (permanent)

- Phone number

\begin{tabular}{lll}
\multirow{2}{*}{$\begin{array}{ll}\text { Tertiary care } \\
\text { hospital }\end{array}$} & Quality operating process & Document no: \\
\cline { 2 - 3 } & Manual of operations & Date of issue: \\
\hline
\end{tabular}

The staff on registration counter will be responsible for validating the information given by the patient and/or their caretaker/relative.

\section{IMPORTANT}

- Check for multiple patient registrations

- Patients can give more than one name and date of birth/ naming date especially non-English speakers

- Always ask for complete name of the patient

- Do not use initials of the patient's name for registration b. Consultation: The healthcare professional should verbally and positively identify the patient prior to treating or examining the patient. If the patient is unable to positively identify themselves, verify the information by asking family member, caretaker or another member who may be accompanying them to the clinic.

The following details should be verbally confirmed/ matched before examining the patient by the consultant.

- Patient's full name

- Patient's date of birth

- Patient's address.

\section{DAY CARE PATIENTS}

All patients attending in day care for day treatment MUST wear an identity bracelet for safety purposes. The ID band should be applied to the patient on arrival of the patient by assigned nurse.

\begin{tabular}{lll}
\multirow{2}{*}{$\begin{array}{l}\text { Tertiary care } \\
\text { hospital }\end{array}$} & Quality operating process & Document no: \\
\cline { 2 - 3 } & Manual of operations & Date of issue: \\
& Patient identification policy & \\
\hline
\end{tabular}

\section{BLOOD TRANSFUSION}

It is applicable to all nurses and doctors initiating the transfusion of blood and blood products.

The bedside check is an important step in preventing transfusion error. Two practitioners are responsible for positive patient identification.

- Check verbally

- Check ID band

In case of any doubt regarding patient's identity - Do not give blood.

\section{RADIOLOGY}

It is the ultimate responsibility of the technician to ensure that the correct patient is being examined according to the request that has been made.

If the patient details stated on the request form are incomplete or have not been completed correctly, further information must be obtained before an exposure is performed. The exposure must not be performed until the patient's identification has been verified.

\section{Situations where a Patient may not Wear an ID Band}

- If the band causes skin irritation

- If patient removes ID band

If a patient is unable to wear an ID band due to their clinical condition an alternative method of identification, where possible, should be sought, e.g. label affixed to the patient's gown. 


\begin{tabular}{lll}
\hline \multirow{2}{*}{$\begin{array}{l}\text { Tertiary care } \\
\text { hospital }\end{array}$} & Quality operating process & Document no: \\
\cline { 2 - 3 } & Manual of operations & Date of issue: \\
\hline
\end{tabular}

\section{INCIDENT REPORTING SYSTEM}

Any staff member if finds any deviation from this policy must make an incident report and submit it to his/her superior or In-charge of the respective area.

This would include an incident that has occurred as a result of misidentification and also 'near miss' situations where the error has been detected before an incident has taken place.

\section{Action in the Event of an Error}

- The root cause analysis of the event should be done. The report should be submitted to the administrator within 48 hours of the event.

- The required corrective and preventive actions must be taken to prevent future occurrence of such events. Action may include further training, etc.

- Any actual error must be explained to the patient involved and, if the patient consents, to relatives/careers. The patient should be kept fully appraised of the action the staff have taken and any necessary future action.

\section{HOW TO MONITOR COMPLIANCE TO THE POLICY?}

Monitoring of compliance with this policy can be carried through audits and therefore audits will be organized by the administrative department on quarterly basis/by annually in all clinical areas.

- The audit will investigate:

- The number and percentage of patients wearing ID bands

- The accuracy and reliability of the information included on them

- The reasons why patients may not be wearing wristbands

- The efficacy of alternative arrangements deployed when patients are not able to wear ID bands

- The number of safety incidents reported which relate to ID bands in any way.

\begin{tabular}{lll}
\multirow{2}{*}{$\begin{array}{l}\text { Tertiary care } \\
\text { hospital }\end{array}$} & Quality operating process & Document no: \\
\cline { 2 - 3 } & Manual of operations & Date of issue: \\
& Patient identification policy & \\
\hline
\end{tabular}

Apart from regular audit, at local level all incidents should be discussed with the team at team meetings following an incident and where appropriate a review undertaken, identifying any actions necessary to prevent future errors.

- Do's and Don'ts to prevent misidentification. ${ }^{21}$

- Do identify the patient correctly on admission.
- Do ensure that you have the full birth-registered name of the patient. The initials of patient's name should never be used for patient identification

- Do check again with the patient that all the details are correct, when you apply an ID band.

- Do regularly check the legibility of ID bands. Replace any bands, in which any part of the patient's details has become illegible.

- Do always check the details of patients even if you think you know them well.

- Do double check verbally and physically that the details of a patient matches the details on a fully completed request form, especially if another member of the healthcare team has completed the form.

- Do label samples taken from the patient straight away. The safest way is to label the bottles after the sample has been taken and before leaving the patient's bedside.

\section{Do Not}

- Do not read the patients details to them and allow them to passively agree with you. Ask the patient to give you their full details.

- Do not take blood from a patient without checking the patients' details against a fully completed request form.

- Do not label a sample bottle before you take blood. You may get distracted before you have completed the task.

- Do not perform two tasks at the same time, e.g. taking blood from several patients and labelling them afterwards or filling out request forms for several patients at the same time.

- Do not perform tasks remotely from the patient if at all possible. Try to fill out request forms and complete tasks at the patient's bedside.

\section{Recommendations}

In a tertiary care hospitals with mandates of education, research and patient care as its mission. Improving patient safety is a multi faceted tasks which will require involvement of all the players in a healthcare system.

The special emphasis shall be on the following points:

- The role of hospital leadership in making patient safety a priority

- The need to improve reporting to capture the extent and causes of adverse events

- The role of a 'just' organizational culture in learning from mistakes

- The need for training and education for professionals, patients and families.

- Next steps is to find out what causes adverse events and how to prevent them. 
One of the ways to avoid human error is to reduce reliance on short-term memory. This limited cognition should be used to perform only essential tasks. Checklists, guidelines and reminders are successful tools for workers to ensure better patient communication. Simple techniques like identification by color coding or eliminating drugs that sound or look alike have proved to be effective in patient safety. Just formulations of guidelines, polices are not enough to have patient safety culture in an organization. Implementation and further monitoring is vital.

\section{Strengths}

Our study has several strengths, like we had used validated checklist. The checklist formulation included individuals from different professions who are directly or indirectly related to the hospital services.

\section{Limitations}

Our study has few limitations like, this was a study conducted in a tertiary care hospital hence the results of this study cannot be generalized to secondary and primary care hospital.

\section{REFERENCES}

1. Wong, Jiahui Wong BH. Strategies for hospitals to improve patient safety: a review of the litreture. The Change Foundation; 2004. p. 1-40.

2. Emanuel L, Berwick D, Conway J, Combes J, Hatlie M, Leape L, et al. What Exactly Is Patient Safety? Advances in Patient Safety: New Directions and Alternative Approaches 2008;1A:1-18.

3. Bates DW, Cullen DJ, LAP NL. Incidence of adverse drug events and potential adverse drug events. JAMA 1995;274(1):29-34.

4. Classen DC, Pestotnik SL. Adverse drug events in hospitalized patients. JAMA 1997;27(4):301-306.
5. Christopher, Error DL. Government reported by the U. Medical errors in the USA: human or systemic? Lancet 2011 Apr 16;377(9774):1289.

6. Pascale Carayon University of Wisconsin - Madison KEWU of WH and C. Patient Safety: The Role of Human Factors and System Engineering. Stud Health Technol Inform 2011; 23-46.

7. Morey JC, Simon R, Jay GD, Wears RL, Salisbury M, Dukes KA, et al. Quality of Care Error Reduction and Performance Improvement in the Emergency Department through Formal Teamwork Training: Evaluation Results of the MedTeams Project 1553-1581.

8. Management IP, Letens G. Transformational Change: A Multilevel Evolutionary Perspective Geert Letens; 2009.

9. Carayon P, Gurses AP. Chapter 30. Nursing Workload and Patient Safety - A Human Factors Engineering Perspective 2000;(1).

10. Baker DP, Day R, Salas E. Teamwork as an essential component of high-reliability organizations. Health services research 2006 Aug;41(4 Pt 2):1576-1598.

11. World Health Organization. Human Factors in Patient Safety Review of Topics and Tools; 2009. p. 1-55.

12. The Joint Commission. National Patient Safety Goals Effective; 2014 Jan:1-17.

13. Lucas A. Positive Identification of Patients Royal United Hospital Bath, NHS. 2010 Feb:1-19.

14. Ogden G. Trust policy and procedures for patient identification, Derby Hospital NHS. 2013;(September 2010):1-20.

15. Norris B, Sevdalis N, Bothwell S, Ranger C, Rose T. The role of patient identification in patient safety 2005(July).

16. Policy and procedure on Identification of Patients, Nottinghamshire Healthcare NHS 2013;(4).

17. Sheahan S. Patient Identification Policy \& Procedure, Mid Western Regional Hospital, Limerick; 2010. p. 1-11.

18. Patient Identification Policy, Cardiff and vale university Health Board; 2015:1-13

19. Stott R. Patient Identification Policy, Doncaster and Bassetlaw Hospitals, NHS. 2013;7(April 2013):1-32.

20. Patient Identification Policy, Gateshead Health NHS. 2014;1-33.

21. Alison Kelly DCN. Patient identification policy, University Hospital of South Manchester, NHS. 2011. p. 1-26. 